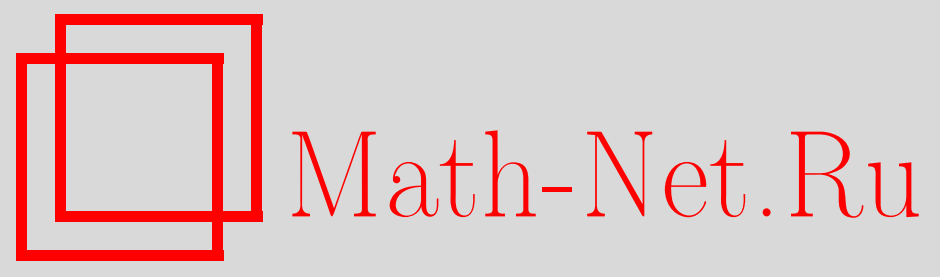

И. А. Шейпак, Нетривиальные фракталы на плоскости и линейные операторы с совместным спектральным радиусом 1, Матем. заметки, 1998, том 63, выпуск 5, 797-800

DOI: https://doi.org/10.4213/mzm1349

Использование Общероссийского математического портала Math-Net.Ru подразумевает, что вы прочитали и согласны с пользовательским соглашением http://www. mathnet.ru/rus/agreement

Параметры загрузки:

IP: 35.173 .219 .149

26 апреля 2023 г., 13:58:06

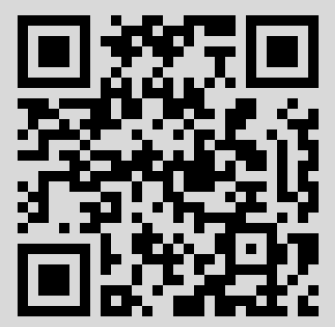




\section{НЕТРИВИАЛЬНЫЕ ФРАКТАЛЫ НА ПЛОСКОСТИ И ЛИНЕЙНЫЕ ОПЕРАТОРЫ С СОВМЕСТНЫМ СПЕКТРАЛЬНЫМ РАДИУСОМ 1}

\section{И. А. Шейпак}

Введение. Нетривиальным фракталом в пространстве $\mathbb{R}^{n}$ мы будем назьвать компактное множество в $\mathbb{R}^{n}$, у которого хаусдорфова размерность $D_{H} \in(n-1, n][1]$.

Совместным спектральным радиусом операторов $A_{1}, \ldots, A_{k} \in L\left(\mathbb{R}^{n}\right)$ согласно работе [2] назьвается число

$$
\widehat{r}\left(A_{1}, \ldots A_{k}\right)=\lim _{m \rightarrow \infty} \max _{\sigma}\left\|A_{\sigma(1)} \cdots A_{\sigma(m)}\right\|^{1 / m}
$$

где максимум берется по всевозможным функциям $\sigma:\{1, \ldots, m\} \rightarrow\{1, \ldots, k\}, L(X)$ обозначает пространство линейных непрерывных операторов в линейном метрическом пространстве $X$.

Отметим, что понятие совместного спектрального радиуса впервые появилось в работе [3]. В [2] были изучены свойства инвариантных тел операторов $A_{1}, \ldots, A_{k} \in L\left(\mathbb{R}^{n}\right)$ и был дан алгоритм поиска совместного спектрального радиуса этих операторов. Рассмотренные примеры инвариантных множеств на плоскости имеют размерность (как топологическую, так и хаусдорфову) или 0 или 1. Целью настоящей заметки является построение класса операторов, инвариантные множества которых имеют дробную хаусдорфову размерность.

1. Инвариантные множества сжимающих отображений. Рассмотрим линейноеполное метрическое пространство $X$ с метрикой $d$. Для отображения $F: X \rightarrow X$ определим его липшицеву константу

$$
\operatorname{Lip} F=\sup _{x \neq y} \frac{d(F(x), F(y))}{d(x, y)} .
$$

Отображение $F$ липшицево, если $\operatorname{Lip} F<\infty$, и слимающее, если $\operatorname{Lip} F<1$.

Введем в $X$ конечньй набор сжимающих отображений $W=\left\{S_{1}, \ldots, S_{N}\right\}$. Для произвольного $A \in X$ положим $\mathscr{S}(A)=\bigcup_{i=1}^{N} S_{i}(A)$. Под $S_{i}(A)$ понимается множество $S_{i}(A)=\left\{S_{i}(x) \mid x \in A\right\}$. Положим $W^{0}(A)=A, W^{1}(A)=W(A), W^{p}(A)=W\left(W^{p-1}\right)$ при $p \geqslant 2$. Согласно [4] дадим следующее

ОПРЕДЕЛЕНИЕ 1. А инвариантно по отношению $\kappa W$, если $A=W(A)$.

В работе [4] доказано следующее свойство сжимающих отображений в полном метрическом пространстве.

Теорема 1. 1) Существует единственное замкнутое ограниченное мнохество $K$, инвариантное по отношению $\kappa W$. (Это мнохество $K$ называют аттрактором системы схимающ,х отобрахсений $W$.) При әтом $K=\bigcup_{i=1}^{N} K_{i}$. $\oint a$.

2) Если $A$ - непустое ограниченное мнохество, то $W^{p}(A) \rightarrow K$ в метрике Хаусдор-

Метрика Хаусдорфа вводится на классе $\mathscr{B}$ непустых замкнутых ограниченных подмножеств пространства $X$ следующим образом:

где $d(x, A)=\inf \{d(x, a): a \in A\}$.

$$
\delta(A, B)=\sup \{d(a, B), d(b, A): a \in A, b \in B\}
$$

Работа выполнена при поддержке Российского фонда фундаментальных исследований, гранты № 96-01-01292 и № 96-15-96091.

(C) И. А. ШЕЙпАк

1998 
Эта теорема позволяет находить сжимающие отображения для уже данных фрактальньх объектов в $\mathbb{R}, \mathbb{R}^{2}$ и $\mathbb{R}^{3}$. Например, легко видеть, что канторово множество на отрезке $[0,1]$ является инвариантньм относительно следующей системы аффинных преобразований $W=\left\{S_{1}, S_{2}\right\}$, где $S_{1}(x)=x / 3, S_{2}(x)=x / 3+2 / 3$. Треугольник Серпинского, вершины которого имеют координаты $(0,0),(0,1),(1,0)$, является аттрактором системы из трех аффинных преобразований $W=\left\{S_{1}, S_{2}, S_{3}\right\}$, где

$$
\begin{gathered}
S_{1}(x, y)=\left(\begin{array}{cc}
0.5 & 0 \\
0 & 0.5
\end{array}\right)\left(\begin{array}{l}
x \\
y
\end{array}\right), \quad S_{2}(x, y)=\left(\begin{array}{cc}
0.5 & 0 \\
0 & 0.5
\end{array}\right)\left(\begin{array}{l}
x \\
y
\end{array}\right)+\left(\begin{array}{c}
0.5 \\
0
\end{array}\right) \\
S_{3}(x, y)=\left(\begin{array}{cc}
0.5 & 0 \\
0 & 0.5
\end{array}\right)\left(\begin{array}{l}
x \\
y
\end{array}\right)+\left(\begin{array}{c}
0 \\
0.5
\end{array}\right) .
\end{gathered}
$$

Основываясь на принципах, изложенных в [4], в серии работ [5]-[7] построен алгоритм, позволяющий или легко конструировать фракталы, или по заданному фракталу найти аффинные преобразования, относительно которых данный фрактал инвариантен. Дальнейшее развитие этих алгоритмов нашло широкое применение в теории сжатия компьютерных изображений.

ЗАмеч АниЕ. Так как отображения сжимающие, в качестве начальной точки можно брать произвольную точку.

Легко видеть, что рассмотренные преобразования являются преобразованиями подобия, которые разлагаются в композицию растяжения (сжатия), поворота и сдвига. Рассмотрим только комбинацию гомотетии и поворота. Каждое аффинное отображение представимо в виде $S=\widehat{S}+a$, где $S$ - линейная часть, а $a$ - вектор сдвига. Очевидно, что в каждом из предложенных примеров семейство операторов $\left\{\widehat{S}_{i}\right\}_{i=1}^{i=N}$ имеет совместньй спектральный радиус, строго меньший 1 . Для множества Кантора $\widehat{r}=1 / 3$, для треугольника Серпинского $\widehat{r}=0.5$. В дальнейшем мы будем рассматривать только аффиинные преобразования.

2. Инвариантные множества семейства операторов с совместным спектральным радиусом, равным 1. Для полноты изложения нам понадобятся понятия и результаты работы [2]. Пусть дан набор линейных операторов $A_{1}, \ldots, A_{k} \in L\left(\mathbb{R}^{n}\right)$. Пусть $x \in \mathbb{R}^{n} \backslash\{0\}$. Через $\mu(x)$ обозначим орбиту точки $x$ под действием всевозможных композиций операторов $A_{1}, \ldots, A_{k}$, т.e.

$$
\mu(x)=\left\{y \in \mathbb{R}^{n} \mid \exists m \in \mathbb{N}: y=A_{\alpha_{1}} \cdots A_{\alpha_{m}}(x), \alpha_{i} \in\{1, \ldots, k\}, i=1, \ldots, m\right\} .
$$

Обозначим через $\mu^{\prime}(x)$ множество предельных точек $\mu(x)$ :

$$
\begin{gathered}
\mu^{\prime}(x)=\left\{y \in \mathbb{R}^{n} \mid \exists m_{i} \in \mathbb{N}, i=1,2, \cdots:\left\|A_{\alpha_{m_{1}}} \cdots A_{\alpha_{m_{i}}}(x)-y\right\| \rightarrow 0, i \rightarrow \infty,\right. \\
\left.\alpha_{m_{j}} \in\{1, \ldots, k\}, j=1, \ldots, i\right\} .
\end{gathered}
$$

Рассмотрим $M(x)$ - замькание выпуклой оболочки множества $\mu^{\prime}(x)$, т.е. $M(x)=\overline{\operatorname{Conv}\left(\mu^{\prime}(x)\right)}$, и оператор $\bar{A}$ на множестве выпуклых тел (т.е. выпуклых компактов с непустой внутренностью), действующий по правилу

$$
\bar{A} M=\operatorname{Conv}\left(\bigcup_{i=1}^{k} A_{i} M\right) .
$$

ОПРЕДЕЛЕНИЕ 2. Инвариантным множеством или инвариантным телом операторов $A_{1}, \ldots, A_{k} \in L\left(\mathbb{R}^{n}\right)$ назьвается выпуклое тело $M$ такое, что $\bar{A} M=\lambda M$ для некоторого $\lambda \in \mathbb{R}_{+}$.

Верна следующая теорема.

Tеорема 2 [2]. Если операторь $A_{1}, \ldots, A_{k} \in L\left(\mathbb{R}^{n}\right)$ не имеют общего инвариантного подпространства (в работе [2] такие операторы называлотся операторами общего полохения) и таковы, что $\widehat{r}\left(A_{1}, \ldots, A_{k}\right)=1$ u $x \in \mathbb{R}^{n} \backslash\{0\}$, то

1) $\mu(x)$ - ограниченное мнохсество;

2) $\mu^{\prime}(x)$ содержит хотя бы один ненулевой элемент;

3) $\operatorname{dim} M(x)=n$;

4) $\bar{A} M(x)=M(x)$;

5) $\mu^{\prime}(x)=\bigcup_{i=1}^{i=k} A_{i}\left(\mu^{\prime}(x)\right)$. 
3. Примеры. В [2] приведено много примеров инвариантных выпуклых тел $M(x)$. В силу 5$)$ теоремы 2 (cp. с 1 ) теоремы 1 ) множество $\mu^{\prime}(x)$ тоже можно считать инвариантным множеством семейства операторов $A_{1}, \ldots, A_{k} \in L\left(\mathbb{R}^{n}\right)$ с совместным спектральным радиусом, равным 1 , но только в смысле определения 1 , а не определения 2 .

ПримеР 1. Рассмотрим поворот в $\mathbb{R}^{2}$ на угол $\alpha$. Если $\alpha / \pi \notin \mathbb{Q}$, то $\mu^{\prime}(x)$ будет окружностью независимо от начальной точки $\left(x_{0}, y_{0}\right)$. Хаусдорфова размерность в этом случае равна 1 . Если брать два оператора, осуществляющих повороты в $\mathbb{R}^{2}$, и один из поворотов не сравним рационально с $\pi$, то множество $\mu^{\prime}(x)$ будет тем же самым.

ПримеР 2. Если $\alpha / \pi=p / q$, где $p / q$ - несократимая дробь, то $\mu^{\prime}(x)$ представляет собой вершины правильного $n$-угольника, $n=2 q$ при нечетных $p$ и $n=q$ при четных $p$. Хаусдорфова размерность этого множества равна 0 .

4. Инвариантные множества с дробной размерностью. Займемся теперь конструкцией совокупности линейных операторов, имеющих совместный единичный спектральньй радиус, но инвариантное множество которых может иметь любую хаусдорфову размерность из промежутка $[0,2]$.

При проективном преобразовании вектору $(x, y)$ в $\mathbb{R}^{2}$ соответствует вектор $(x, y, 1)$. Соответственно аффинному преобразованию в $\mathbb{R}^{2}$

$$
S(x, y)=\left(\begin{array}{ll}
a & b \\
c & d
\end{array}\right)\left(\begin{array}{l}
x \\
y
\end{array}\right)+\left(\begin{array}{l}
e \\
f
\end{array}\right)
$$

отвечает линейное преобразование в $\mathbb{R}^{3}$ :

$$
A(x, y, 1)=\left(\begin{array}{lll}
a & b & e \\
c & d & f \\
0 & 0 & 1
\end{array}\right)\left(\begin{array}{l}
x \\
y \\
1
\end{array}\right)
$$

Таким образом, набору аффинных сжимающих отображений $A_{1}, \ldots, A_{k}$ в $\mathbb{R}^{2}$, где

$$
A_{i}(x, y)=\left(\begin{array}{cc}
a_{i} & b_{i} \\
c_{i} & d_{i}
\end{array}\right)\left(\begin{array}{c}
x \\
y
\end{array}\right)+\left(\begin{array}{c}
e_{i} \\
f_{i}
\end{array}\right)
$$

соответствует набор линейных операторов в $\mathbb{R}^{3}$ с совместным спектральным радиусом, равным 1 . Очевидно, что эту схему легко продолжить на пару сжимающих афффинных преобразований в $\mathbb{R}^{n}-$ линейных преобразований в $\mathbb{R}^{n+1}$ с совместным спектральным радиусом $\widehat{r}=1$. Но в этом случае все операторы имеют общее инвариантное одномерное подпространство; поэтому инвариантное множество $\mu^{\prime}(x)$ принадлежит $\mathbb{R}^{n}$. На основании вьшесказанного справедлива следующая

Теорема 3. Если линейные операторы с матрицами

$$
A_{i}=\left(\begin{array}{ccc}
a_{i} & b_{i} & e_{i} \\
c_{i} & d_{i} & d_{i} \\
0 & 0 & 1
\end{array}\right), \quad i=1, \ldots, n
$$

maковы, что

$$
S_{i}=\left(\begin{array}{ll}
a_{i} & b_{i} \\
c_{i} & d_{i}
\end{array}\right)
$$

порождают сжимающее отображсение в $\mathbb{R}^{2}$, то

1) существует единственное мнохсество $\mu^{\prime}$, являющееся инвариантным мнохсеством семейства операторов $A_{i}, i=1, \ldots, n$, а именно, $\mu^{\prime}=\bigcup_{i=1}^{i=n} A_{i}\left(\mu^{\prime}\right) ;$ это мнохсество не зависит от начальной точки х и принадлехит плоскости, определяемой условием $z=1$;

2) для любого ограниченного непустого мнохества $M W^{p}(M) \rightarrow \mu^{\prime}$ в метрике Хаусдорфа. 
Приведем пример набора двух операторов, позволяющий конструировать инвариантные множества с любой дробной размерностью:

$$
\begin{aligned}
& A_{1}(x, y, 1)=\left(\begin{array}{ccc}
\frac{1}{2} & -\frac{1}{2} \operatorname{tg} \varphi & 0 \\
\frac{1}{2} \operatorname{tg} \varphi & \frac{1}{2} & 0 \\
0 & 0 & 1
\end{array}\right)\left(\begin{array}{l}
x \\
y \\
1
\end{array}\right), \\
& A_{2}(x, y, 1)=\left(\begin{array}{ccc}
\frac{1}{2} & \frac{1}{2} \operatorname{tg} \varphi & \frac{1}{2} \\
-\frac{1}{2} \operatorname{tg} \varphi & \frac{1}{2} & \frac{1}{2} \operatorname{tg} \varphi \\
0 & 0 & 1
\end{array}\right)\left(\begin{array}{l}
x \\
y \\
1
\end{array}\right),
\end{aligned}
$$

Хаусдорфова размерность множества $\mu^{\prime}$ определяется по формуле

$$
\operatorname{dim}_{H} \mu^{\prime}=\frac{\ln 2}{\ln 2 \cos \varphi} .
$$

При $\varphi=0$ инвариантное множество представляет собой отрезок $[0,1]$ (размерность равна 1 ). При $\varphi \geqslant \pi / 4$ имеем $\operatorname{dim}_{H} \mu^{\prime}(x)=2$. Примеры множеств при $\varphi=\pi / 6, \varphi=2 \pi / 9$ и $\varphi=\pi / 4$ приведены на рис. 1,2 и 3 соответственно.

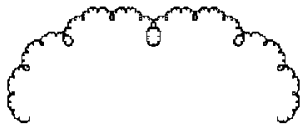

Рис. 1

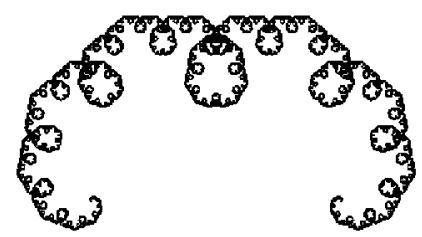

Рис. 2

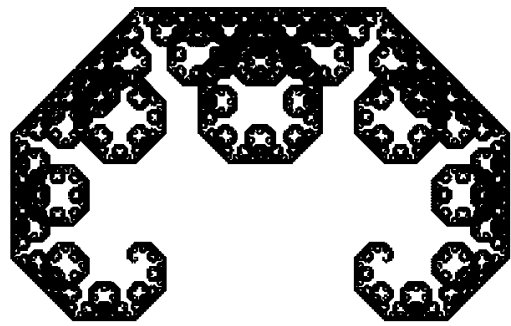

Рис. 3

Обобщая теорему 3 на случай операторов, действующих в $\mathbb{R}^{n+1}$ и имеющих вид

$$
A_{i}=\left(\begin{array}{cc}
S_{i} & E_{i} \\
0 & 1
\end{array}\right)
$$

где $E_{i}$ есть $n$-мерный вектор, а $S_{i}$ является $(n \times n)$-матрицей, надо потребовать, чтобы $S_{i}$ задавала сжимающее отображение в $\mathbb{R}^{n}$. Множество $\mu^{\prime}$ в этом случае будет принадлежать $n$-мерной гиперплоскости в $\mathbb{R}^{n+1}$. Также можно сконструировать множествос любой хаусдорфовой размерностью из промежутка $[0, n+1]$.

\section{СПИСОК ЦИТИРОВАННОЙ ЛИТЕРАТУРЫ}

1. Mandelbrot B. Fractals, Form, Chance, and Dimension. San Francisco: Freeman, 1977. 2. Протасов В. Ю. // Фундамент. и прикл. матем. 1996. Т. 2. №1. С. 205-231. 3. Rota G. C., Strang G. // Indag. Math. 1960. V. 63. P. 379-381. 4. Hutchinson J. E. // Indiana Univ. Math. J. 1981. V. 30. P. 713-747. 5. Barnsley M. F., Demko S. // Proc. Roy. Soc. London. Ser. A. 1985. V. 399. P. 243-275. 6. Barnsley M. F., Sloan A. D. // Byte Mag. 1988. P. 215-223. 7. Barnsley M. F. Fractals Everywhere. London: Acad. Press, 1988. 